\title{
Correlated-electron description of the photophysics of thin films of $\pi$-conjugated polymers
}

\author{
D. Psiachos and S. Mazumdar \\ Department of Physics, University of Arizona Tucson, AZ 85721
}

(Dated: November 17, 2018)

\begin{abstract}
We extend Mulliken's theory of ground state charge transfer in a donor-acceptor complex to excited state charge transfer between pairs of identical $\pi$-conjugated oligomers, one of which is in the optically excited state and the other in the ground state, leading to the formation of a chargetransfer exciton. Within our theory, optical absorptions from the charge-transfer exciton should include a low energy intermolecular charge-transfer excitation, as well as distinct intramolecular excitations from both the neutral delocalized exciton component and the Coulombically bound polaronpair component of the charge-transfer exciton. We report high order configuration-interaction calculations for pairs of oligomers of poly-paraphenylenevinylene (PPV) that go beyond our previous single configuration-interaction calculation and find all five excited state absorptions predicted using heuristic arguments based on the Mulliken concept. Our calculated excited state absorption spectrum exhibits strong qualitative agreement with the complete wavelength-dependent ultrafast photoinduced absorption in films of PPV derivatives, suggesting that a significant fraction of the photoinduced absorption here is from the charge-transfer exciton. We make detailed comparisons to experiments, and a testable experimental prediction.
\end{abstract}

PACS numbers: 42.70.Jk, 71.35.-y, 78.20.Bh, 78.30.Jw 


\section{INTRODUCTION}

It is now generally recognized that interchain interactions play a strong role in the photophysics of thin films of $\pi$-conjugated polymers (PCPs) $1,2,3,4,5,6,7,8,9,10,11,12,13,14,15,16,17$ While the primary photoexcitation in dilute solutions of PCPs, which contain widely separated single strands, is to the optical exciton, interchain species other than the intrachain optical exciton are generated in films. Since the original demonstration of interchain species in films of poly(2-methoxy,5-(2'-ethyl-hexyloxy)1,4 paraphenylenevinylene) (MEH-PPV) by Rothberg and collaborators, $\frac{1}{-}$ a variety of complex optical phenomena in many other PCPs have been ascribed to interchain interactions. Experiments that indicate the formation of interchain species upon photoexcitation include, (i) photoluminescence (PL) at longer wavelength relative to solutions, with smaller quantum efficiency, $, 2,2,3,4,5,6,11$ (ii) delayed PL lasting until milliseconds, and its quenching by an electric field,,$\stackrel{1,2,14,15}{1}$ and (iii) additional photoinduced absorptions (PAs) in ultrafast spectroscopy of films over and above those observed in solutions. $1,12,13$

Three different kinds of interchain species can in principle occur in PCPs. These include, (i) neutral exciton delocalized over multiple chains, as in an aggregate, $11,18,19,20$ (ii) completely ionic polaron-pair, with positively and negatively charged neighboring chains that are Coulombically bound, $, 2, \underline{4}$, and (iii) the charge-transfer (CT) exciton, which is a quantum mechanical superposition of the delocalized neutral exciton and the ionic polaron-pair that is obtained in the presence of significant interchain electron hopping. ${ }^{4,21}$ All three kinds of interchain species have been proposed to explain experiments in real systems. PL at wavelengths longer than in solutions with weaker quantum efficiency can be explained using the H-aggregate scenario, wherein absorption is to the highest state in the exciton band and emission occurs from the lowest state in the band. $\underline{11} \underline{19}$ On the other hand, PA in films was originally thought to be from a polaron-pair state. $\underline{1}^{\underline{4}}$ Very recently, Wang et al. $\underline{\underline{21}}$ have claimed that the detailed features of the PA in films $\frac{12,13}{13}$ can be understood only within the CT exciton picture. According to Wang et al. a significant fraction of both PL and PA occur from the CT exciton. The calculations of the PA in Reference 21 were based on the single configuration-interaction (SCI) approximation, and were able to reproduce only a limited low energy region of the experimental PA spectra. ${ }^{12,13}$ In the present work we go beyond SCI and perform quadruple CI and multiple reference singles and double CI (MRS- 
DCI) calculations $22, \underline{23}$ of excited state absorptions in interacting pairs of PPV oligomers to understand the PA spectra over the complete experimental energy range. Our calculated PA spectra, originating from the CT exciton, exhibit strong qualitative agreement with the experimental PA spectra in films of PPV derivatives, $\frac{12}{2}$ in spite of the short lengths of the oligomers for which the many-body calculations could be performed.

It is important for what follows that we clearly state the difference between PA in solutions and films. Both solutions and films show absorptions labeled $\mathrm{PA}_{1}$ and $\mathrm{PA}_{2}$. The lower energy $\mathrm{PA}_{1}$ exhibits a peak at $\sim 1 \mathrm{eV}$ and the higher energy $\mathrm{PA}_{2}$ occurs at $\sim 1.3-1.4 \mathrm{eV}$. Two distinct additional PAs, $\mathrm{P}_{1}$ at $\sim 0.4 \mathrm{eV}$ and $\mathrm{P}_{2}$ close to, but distinct from $\mathrm{PA}_{2}$, are seen in films $\underline{12,13}$ Whether or not $\mathrm{P}_{1}$ and $\mathrm{P}_{2}$ arise from free polarons (generated by exciton dissociation) or from a bound interchain species has been controversial in the past (see discussions in reference 21). Recent experiments have found similar behavior in heterostructures consisting of donor and acceptor PCPs, $\underline{24}, \underline{25}$ or of PCPs and finite molecular acceptors $\underline{26}$ Identifying the nature of the dominant intermolecular species, and determining their photophysics is clearly important.

In section II we discuss our theoretical model and the methods we use. In view of the highly correlated natures of the two-chain wavefunctions, and the complexity of the phenomenon of excited state charge transfer that is only beginning to be understood, we present in section III heuristic physical descriptions of all photoexcitations that are possible from the CT exciton. This discussion is an extension of Mulliken's theory of ground state charge transfer between a donor and an acceptor ${ }^{27}$ to excited state charge transfer between identical pairs of molecules, one of which is in the optically excited state. The qualitative arguments make the computational results, presented in section IV, understandable. We present our conclusions, along with comparisons to experiments, in section $\mathrm{V}$.

\section{THEORETICAL MODEL AND METHODS}

As in Reference 21, we consider pairs of capped PPV oligomers $4 \mathrm{~nm}$ apart, stacked cofacially. The sophisticated many-body approaches used here require enormous Hamiltonian bases, $\frac{28}{2}$ which necessarily limits us to oligomers that are considerably shorter than those investigated in our previous SCI calculations. ${ }^{21}$ Our calculations here are for pairs of oligomers of length three and four units each. We refer to the individual oligomers as PPV3 and PPV4, respectively. Note that with two such chains these calculations are much larger than the 
previous QCI and MRSDCI calculations of $\mathrm{PA}_{1}$ and $\mathrm{PA}_{2}$ in single chains. 28 Although the actual arrangements of the chromophores deviate from ideal cofacial stacking in the real systems, it is believed that this assumption captures the essential physics of polymer thin films. $., 18,21$ Unlike in 21, our calculations here are only for pairs of oligomers with equal lengths, so that the entire two-chain structure possesses a center of inversion. This is for computational simplification only and does not affect our conclusions. As indicated in our previous work, lack of inversion symmetry makes the CT exciton below the optical exciton weakly optically allowed from the ground state, but the energy locations and strengths of the calculated PAs originating from this interchain species are the same whether or not inversion symmetry exists. 21

We describe our system within an extended two-chain Pariser-Parr-Pople $\pi$-electron Hamiltonian $^{29,30} H=H_{\text {intra }}+H_{\text {inter }}$, where $H_{\text {intra }}$ and $H_{\text {inter }}$ correspond to intra- and interchain components, respectively. The two components of $H$ are written as,

$$
\begin{aligned}
H_{\text {intra }}=- & \sum_{\mu\langle i j\rangle, \sigma} t_{i j}\left(c_{\mu, i, \sigma}^{\dagger} c_{\mu, j, \sigma}+\text { H.C. }\right)+U \sum_{\mu, i} n_{\mu, i, \uparrow} n_{\mu, i, \downarrow} \\
& +\sum_{\mu, i<j} V_{i j}\left(n_{\mu, i}-1\right)\left(n_{\mu, j}-1\right)
\end{aligned}
$$

and,

$$
\begin{aligned}
H_{\text {inter }}^{1 e} & =-t_{\perp} \sum_{\mu<\mu^{\prime}, i, \sigma}\left(c_{\mu, i, \sigma}^{\dagger} c_{\mu^{\prime}, i, \sigma}+\text { H.C. }\right) \\
& +\frac{1}{2} \sum_{\mu<\mu^{\prime}, i, j} V_{i j}^{\perp}\left(n_{\mu, i}-1\right)\left(n_{\mu^{\prime}, j}-1\right)
\end{aligned}
$$

In the above $c_{\mu, i, \sigma}^{\dagger}$ creates a $\pi$-electron of spin $\sigma$ on carbon atom $i$ of oligomer $\mu(=1,2)$, $n_{\mu, i, \sigma}=c_{\mu, i, \sigma}^{\dagger} c_{\mu, i, \sigma}$ is the number of electrons on atom $i$ of oligomer $\mu$ with spin $\sigma$ and $n_{\mu, i}=\sum_{\sigma} n_{\mu, i, \sigma}$ is the total number of electrons on atom $i$ of oligomer $\mu$. We consider standard intrachain nearest neighbor one-electron hopping integrals $t_{i j}=2.4 \mathrm{eV}$ for phenyl C-C bonds, and 2.2 (2.6) eV for single (double) C-C bonds, respectively. $\underline{\underline{ }} U$ and $V_{i j}$ are the on-site and intrachain intersite Coulomb interactions. $V_{i j}$ are obtained from a modification of the Ohno parametrization 32

$$
V_{i j}=\frac{U}{\kappa \sqrt{1+0.6117 R_{i j}^{2}}}
$$

where $R_{i j}$ is the distance between carbon atoms $i$ and $j$ in $\AA$, and $\kappa$ is the background dielectric constant along the chain direction that takes into account screening effects from 
the inner-shell electrons. Within the standard Ohno parametrization, $U=11.26 \mathrm{eV}$ and $\kappa=1$. We use $U=8 \mathrm{eV}$ and $\kappa=2$. The detailed justification of our parameters has been given elsewhere. $\frac{31}{3}$

As in our previous work, ${ }_{21}^{21}$ interchain hopping is restricted between the closest pairs of carbon atoms; we choose $t_{\perp}=0.1 \mathrm{eV}$. For $V_{i j}^{\perp}$, we choose the same functional forms as in Eq. 3, with the same dielectric screening for the PPV oligomer. As shown before, ${ }^{21}$ the results of the PA calculations are relatively insensitive to variations of the transverse dielectric constant, as long as reasonably realistic values are chosen.

In addition to PA calculations for interacting pairs of neutral chains, we have also calculated the ground state absorption energies of singly-charged three- and four-unit oligomers, for comparison to the PA spectra of neutral systems. These absorption energies can be strongly affected by electron-phonon interactions, which, however, have not been included in our $H_{\text {intra }}$. This is primarily because calculating PA within a model that incorporates both electron-electron and electron-phonon interactions is currently difficult, and the comparison would be meaningless if only one of them was calculated with electron-phonon interactions. We shall nevertheless refer to the charged oligomer absorptions as polaron absorptions.

\section{CHARGE-TRANSFER EXCITONS AND EXCITED STATE ABSORPTION}

We present here a brief review of our earlier work on the CT exciton in interacting PCP chains for the sake of completeness. Following this, we present a diagrammatic description of the excited state absorptions that are expected for the two-chain system, within an extension of the Mulliken theory of charge transfer. ${ }^{27}$

Four distinct basis functions, two neutral and two ionic, are relevant for understanding the correlated eigenstates near the optical edge of PCPs. ${ }^{21}$ These are the excited neutral and charged eigenstates of $H_{\text {intra }}$ for two independent chains. We write the neutral twochain basis functions as product functions of the single-chain $1 \mathrm{~A}_{g}$ ground state and the $1 \mathrm{~B}_{u}$ optical exciton, viz., $|\operatorname{exc} 1\rangle=\left|1 B_{u}\right\rangle_{1}\left|1 A_{g}\right\rangle_{2}$ and $|\operatorname{exc} 2\rangle=\left|1 B_{u}\right\rangle_{2}\left|1 A_{g}\right\rangle_{1}$, respectively, where the suffixes are chain indices. The charged polaron configurations, in their lowest states, are similarly written as $\left|P^{+}\right\rangle_{1}\left|P^{-}\right\rangle_{2}$ and $\left|P^{+}\right\rangle_{2}\left|P^{-}\right\rangle_{1}$. For symmetrically placed chain pairs, and within the SCI, the total Hamiltonian $H$ has four eigenstates: ${ }^{21}$ the even

parity optical exciton, $|E X\rangle=\frac{1}{\sqrt{2}}[|e x c 1\rangle+|e x c 2\rangle]$; the even parity polaron-pair state, 
$|P P\rangle_{+}=\frac{1}{\sqrt{2}}\left[\left|P^{+}\right\rangle_{1}\left|P^{-}\right\rangle_{2}+\left|P^{+}\right\rangle_{2}\left|P^{-}\right\rangle_{1}\right]$; and two distinct charge-transfer excitons, hereafter $|C T X 1\rangle$ and $|C T X 2\rangle$, that are superpositions of the odd parity states $\frac{1}{\sqrt{2}}[|e x c 1\rangle$ $|e x c 2\rangle]$ and $\frac{1}{\sqrt{2}}\left[\left|P^{+}\right\rangle_{1}\left|P^{-}\right\rangle_{2}-\left|P^{+}\right\rangle_{2}\left|P^{-}\right\rangle_{1}\right]$. For the interchain dielectric screening constant less than or equal to the intrachain dielectric screening, the energy ordering of the states ${ }^{21}$ is $|C T X 1\rangle<|E X\rangle<|P P\rangle_{+}<|C T X 2\rangle$.

Within the Mulliken theory, $\underset{27}{2}$ a weakly coupled donor-acceptor system is expected to exhibit a low energy charge-transfer absorption as well as weakly perturbed molecular absorptions. Current pump-probe experiments have very high sensitivity, with $|\Delta T / T| \sim 10^{-4}$ (where $T$ is the transmission of the probe beam without the pump beam, and $\Delta T$ the difference in the transmission with and without the pump) 12 . Under this condition, excited state "molecular absorptions" from $|C T X 1\rangle$ will be visible from both the neutral exciton component as well as the ionic polaron-pair component. We show this schematically in Fig. 1, where we have included all possible excited state absorptions from $|C T X 1\rangle$. Here $\left|E x_{-}\right\rangle$ and $\left|P P_{-}\right\rangle$are the superpositions of the odd parity exciton and polaron-pair configurations mentioned above; we have shown only one member of each symmetry-adapted pair in both cases. Each two-chain configuration is shown as a product state of single-particle configurations, with electrons occupying bonding and antibonding molecular orbitals (MOs), and spin-singlet bonds between the MOs that are singly occupied by electrons.

The charge-transfer excitation from $|C T X 1\rangle$ in Fig. 1 is to the even parity polaronpair state $|P P\rangle_{+}$. This state is reached by intermolecular transfer of an electron from the antibonding (bonding) MO of the molecule in the excited (ground) state of $\left|E x_{-}\right\rangle$to the antibonding (bonding) MO of the molecule in the ground state. The polarization of this transition, in contrast to the intramolecular transitions discussed below, is perpendicular to the plane of the molecules.

Two different intramolecular excitations from the $\left|E x_{-}\right\rangle$component of $|C T X 1\rangle$ are indicated in Fig. 1. The first of these is to a higher energy effective one electron-one hole (1e-1h) excited state that we have labeled as the $\left|m A_{g-}\right\rangle$. The second is an effective two electrontwo hole (2e-2h) excitation labeled $\left|k A_{g-}\right\rangle$. Corresponding to each of these intramolecular excitations of the two-chain CT exciton there exist single-chain excited states that have been extensively discussed in the context of nonlinear absorptions in single chains. $\stackrel{28,31}{2}$ The single-chain $\left|m A_{g}\right\rangle$ and the $\left|k A_{g}\right\rangle$ are the final states corresponding to induced absorptions $\mathrm{PA}_{1}$ and $\mathrm{PA}_{2}$, respectively, from the single-chain $\left|1 B_{u}\right\rangle$ exciton. $\underline{33}, \underline{34}$ The minus subscripts in 


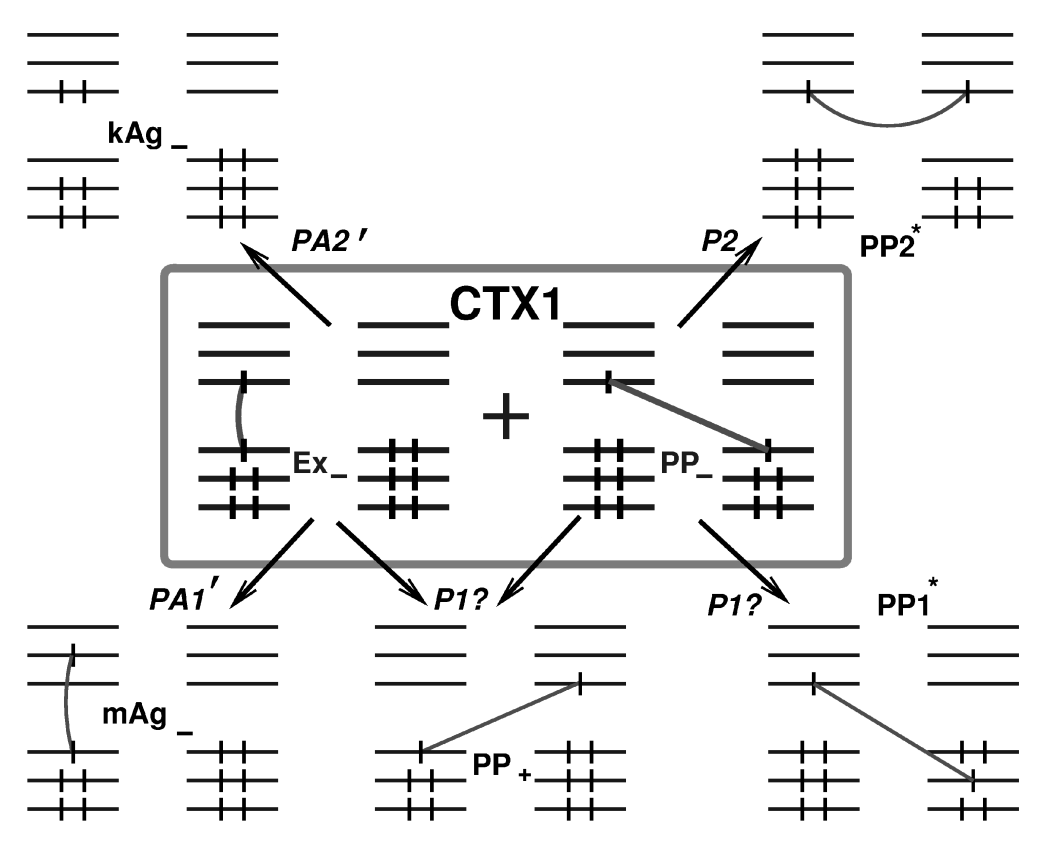

FIG. 1: Schematic of the PAs from the charge-transfer exciton $|C T X 1\rangle$, shown in the box as a superposition of the neutral exciton state $\left|E x_{-}\right\rangle$and the polaron-pair state $\left|P P_{-}\right\rangle$(see text). $\left|P P_{+}\right\rangle$is reached by low energy charge-transfer excitation; $\left|m A g_{-}\right\rangle$and $\left|k A g_{-}\right\rangle$are reached by 1e-1h and 2e-2h intramolecular excitations from the $\left|E x_{-}\right\rangle$component of $|C T X 1\rangle$, giving induced absorptions $\mathrm{PA}_{1}^{\prime}$ and $\mathrm{PA}_{2}^{\prime}$, respectively. $\left|P P 1^{*}\right\rangle$ and $\left|P P 2^{*}\right\rangle$ are reached by $1 \mathrm{e}-1 \mathrm{~h}$ intramolecular excitations from the $\left|P P_{-}\right\rangle$component of $|C T X 1\rangle$ (see text).

$\left|m A_{g-}\right\rangle$ and the $\left|k A_{g-}\right\rangle$ imply that as with the $\left|E x_{-}\right\rangle$, the actual wavefunctions contain odd parity superpositions of two-chain configurations. The $\left|m A_{g-}\right\rangle$ and the $\left|k A_{g-}\right\rangle$ are higher energy CT excitons, and also contain excited polaron-pair components that are not explicitly shown in the Figure. Indeed, each final state wavefunction in Fig. 1 is a superposition of several dominant configurations, only one of which is shown explicitly in the Figure. The others are obtained by applications of mirror-plane and charge-conjugation symmetry operations on the configurations shown. In the case of the $\left|m A_{g-}\right\rangle$ the exciton and excited polaron-pair contributions to the wavefunction were both demonstrated within the SCI in our previous work. ${ }^{21}$

Fig. 1 also indicates intramolecular absorptions from the $\left|P P_{-}\right\rangle$component of $|C T X 1\rangle$. We have labeled the dominant final configurations here as $\left|P P 1^{*}\right\rangle$ and $\left|P P 2^{*}\right\rangle$, which are bound high energy excited polaron-pair configurations. For realistic $t_{\perp}$ and $V_{i j}^{\perp}$, the energies of the excited state transitions from the CT exciton to the polaron-pair states $\left|P P 1^{*}\right\rangle$ and 
$\left|P P 2^{*}\right\rangle$ should be comparable to the ground state absorption energies in charged chains, since the former are exclusively from the charged component of $|C T X 1\rangle$.

\section{COMPUTATIONAL RESULTS}

We find from direct computations of excited state spectra that all of the absorptions indicated in Fig. 1 should indeed be observed. We report results of QCI and MRSDCI calculations on pairs of PPV3 and PPV4 oligomers. In our many-body calculations we have retained all innermost one-electron delocalized bonding and antibonding band levels, and the localized bonding and antibonding bands that occur below and above these.$^{\underline{31}}$ The dimension of the QCI Hamiltonian matrix for pairs of PPV3 oligomers was 1,83,3276. In order to obtain convergence in energies to $0.02 \mathrm{eV}$, the MRSDCI calculations for PPV4 were performed with 54 reference configurations. The overall dimension of the Hamiltonian matrix for the two-chain system in this case was $1,46,8048$. These sizes are at the limit of our computational capability.

In Figs. 2(a) and (b) we have shown our calculated PAs from $|C T X 1\rangle$ for PPV3 and PPV4 oligomers, respectively. The PA energies are given in units of the energy of the twochain optical exciton $|E X\rangle$ in each case. We have also calculated the optical absorption energies from the ground states of the charged single chains $\left(\mathrm{PPV}^{+}\right.$and $\left.\mathrm{PPV}^{+}\right)$. The arrows in the figures denote the charged chain absorption energies, relative to the energy of $|E X\rangle$. The large differences between the charged-chain absorptions and PAs labeled PP1 and PP2 are primarily due to the finite size effects associated with the very short system sizes being probed here, although additional contribution to this difference arises also from interchain Coulomb interactions that influence the energies of the two-chain PAs.

We have calculated the PAs from the two-chain optical exciton $|E X\rangle$. PAs nearly identical in energy to $\mathrm{PA}_{1}^{\prime}$ and $\mathrm{PA}_{2}^{\prime}$ are found, but the PAs labeled CT, PP1 and PP2 are missing in this case, confirming the analysis of Fig. 1. We have also done detailed wavefunction analyses of the final states of all the PAs shown in Fig. 2 to confirm that the dominant configurations in each case are the same as that indicated in the schematic Fig. 1. Thus the final state of the absorption labeled $\mathrm{CT}$ in Fig. 2 is predominantly $\left|P P_{+}\right\rangle$, while the final states of the absorptions labeled PP1 and PP2 are predominantly superposiitons of the polaron-pair configurations shown in Fig. 1. The results of Fig. 2 go considerably beyond 
the SCI studies of reference 21, which had found only the PA labeled $\mathrm{PA}_{1}^{\prime}$ and the low energy charge-transfer absorption. While it is expected that inclusion of higher order CI is essential for detecting the PA labeled $\mathrm{PA}_{2}^{\prime 28}$, it is interesting that this is a requirement also for the PAs labeled PP1 and PP2, in spite of their predominantly 1e-1h characters. A few additional points need to be emphasized. Our earlier SCI calculations were for pairs

of oligomers 2-3 times longer, 21 and from careful finite-size analysis we concluded that the features labeled CT and $\mathrm{PA}_{1}^{\prime}$ will continue to be seen for a broad range of realistic oligomer lengths. While it is not possible to do a similar finite size analysis for the QCI and MRSDCI calculations we report here, based on the previous analysis we are convinced that all PAs in Fig. 2 will similarly be seen at realistic chainlengths. The relative energy separations may, however, be strongly chain-length dependent. Thus from the nature of the final states $\left|P P_{+}\right\rangle$ and $\left|P P_{1}^{*}\right\rangle$ in Fig. 1 it is conceivable that the absorptions labeled CT and PP1 in Fig. 2 are energetically much closer in systems with realistic lengths, especially if electron-phonon interactions play a weak role. Similarly, the relative locations of PP2 and $\mathrm{PA}_{2}^{\prime}$ may also depend on system size. Both of these may have experimental consequences, as discussed in the next section.

\section{DISCUSSIONS AND COMPARISON TO EXPERIMENTS}

Within our theory, photoexcitation in films generates predominantly the optical exciton, along with small amounts of the CT exciton, which is weakly optically allowed due to disorder ${ }^{21} \mathrm{PL}$ is predominantly from the CT exciton, whose neutral component is the same as the lowest forbidden exciton state in a H-aggregate. The mechanism of light emission is therefore the same as in theories emphasizing aggregate formation. ${ }^{11,19,20}$ We believe that PA in films at the earliest times is predominantly from the optical exciton with weak contributions from the CT exciton, while at later times the PA is predominantly from the CT exciton. Recent careful measurements have indicated that the PA labeled $\mathrm{PA}_{1}$ in films exhibit biexponential decay, which would agree with the two-state scenario ${ }^{38}$ Recent ex-

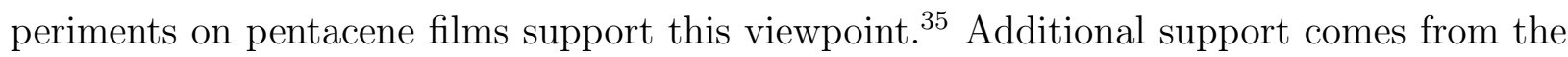
absence of free polarons, as noted in microwave conductivity measurements ${ }^{36}$ and terahertz spectroscopy, 37 in spite of the experimentally observed PA bands $\mathrm{P}_{1}$ and $\mathrm{P}_{2}$ that resemble absorption from charged chains. We believe that an alternate possibility, viz., PL is from the 

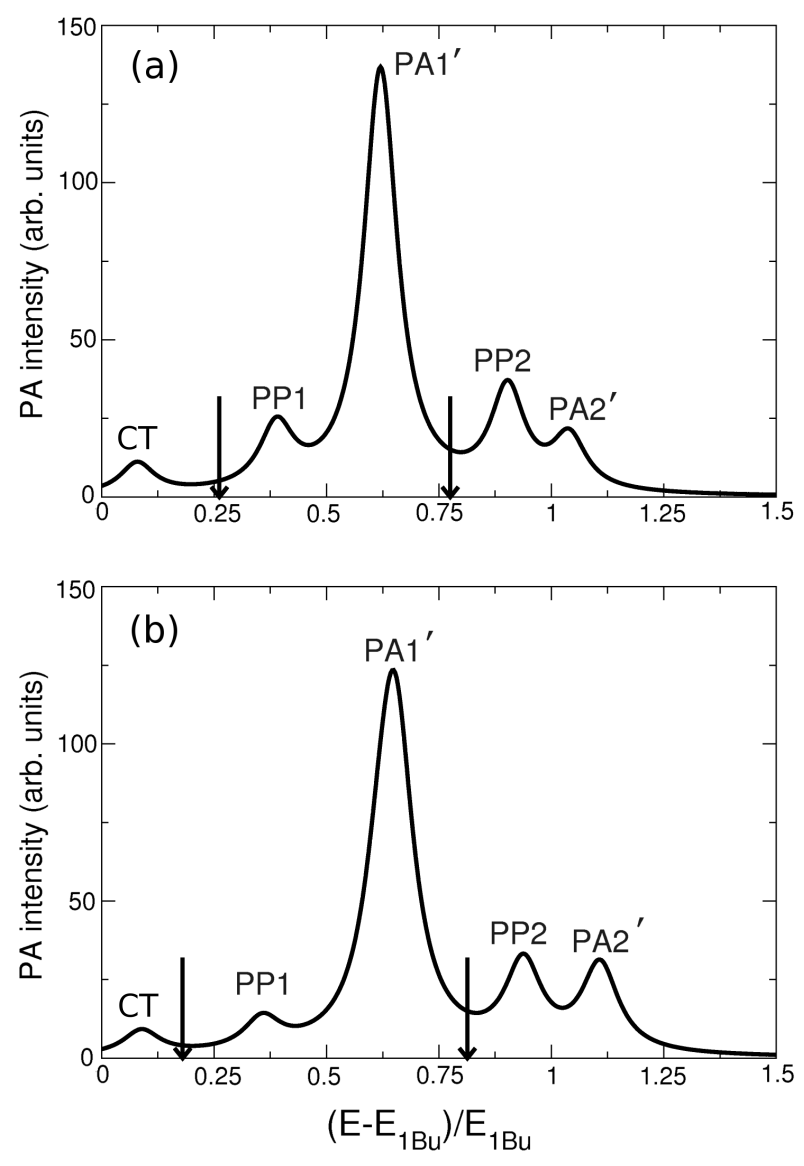

FIG. 2: Calculated PAs from $|C T X 1\rangle$ in pairs of (a) PPV3, and (b) PPV4 oligomers. All energies are relative to that of the neutral optical exciton. Arrows denote energies of absorption from the ground states of charged chains.

exciton but PA is from a bound polaron-pair, is precluded by the absence of room temperature infrared-active vibration (IRAV) in the recent PA experiment. $\stackrel{12}{*}$ Unlike the CT exciton, the polaron-pair wavefunction is not affected by interchain charge-transfer and is asymmetric: i.e., a specific chain is positively charged while the other is negatively charged. The counterpart related by symmetry, with the charges on the two chains reversed, is missing in the polaron-pair, and hence PA even from the bound polaron-pair should be accompanied by strong IRAV. Although strong IRAV has been reported by one research group, $\stackrel{39}{\text { this }}$ results has not been reproduced by any other group.

A key weakness of our theory in Reference 21 was that the PA spectrum could be calculated only for a limited energy range. This is remedied in the present work. Our calculated PA spectra of Fig. 2 are to be compared with the experimental PA spectra of Sheng et 
al. (see Figures 1 and 4 in Reference 12). Remarkable qualitative similarities between the calculated and experimental spectra are found, although from the theoretical spectrum it is difficult to distinguish between the two possible origins of the experimental $\mathrm{P}_{1}$ absorption. From our calculations, the $\mathrm{P}_{1}$ absorption observed experimentally can be due to either the low energy charge-transfer absorption, or the absorption to the $\left|P P 1^{*}\right\rangle$ state. We suggest polarization-dependent experimental measurements to distinguish between these two possibilities: the charge-transfer absorption is polarized perpendicular to the molecular planes, while the absorption labeled PP1 is polarized along the chain direction in the molecular plane. Yet another possibility is that the experimental $P_{1}$ absorption is composed of two underlying absorptions. As pointed out in the previous section, in systems with realistic chain lengths these absorptions will be proximate in energy and can even be very close. In a recent experiment performed by Vardeny et al. on films of PPV derivatives, it has been found that the $\mathrm{P}_{1}$ absorption splits into two distinct peaks under hydrostatic pressure. $\stackrel{38}{ }$ Whether or not these two PAs have different origins, as might be expected from our calculations, or they arise from the splitting of the same absorption under the experimental conditions, is currently not clear, and further experimental work is necessary to clarify this issue.

In conclusion, we have extended the Mulliken theory of charge transfer to the case of excited state charge transfer, to develop a theory of the CT exciton in thin films of $\pi$-conjugated polymers. The central feature of our theory is that PA in films can occur from both the neutral exciton component of the CT exciton as well as from the charged bound polaronpair component. PAs from the exciton components occur at nearly the same wavelengths as those of the single chains that occur in dilute solutions. PAs from the polaron-pair component occur at nearly the wavelengths where ground state absorptions of charged chains occur. Interestingly, the absorptions from the polaron-pair component of the CT exciton are vanishingly weak in SCI calculations and become visible only upon including higher order CI. In addition to these features, the PA spectrum can exhibit a low energy charge-transfer absorption. Whether or not the low energy $\mathrm{P}_{1}$ absorption seen in PA measurements 12 is a superposition of two fundamentally different absorptions is currently not understood, and will require further experimental work. There is no such ambiguity in the case of the absorption we have labeled PP2: this is certainly the origin of the $\mathrm{P}_{2}$ absorption in the experiment $\underline{12}$ Note, however, that the relative energies of PAs labeled PP2 and $\mathrm{PA}_{2}^{\prime}$ in real systems can be slightly different from those predicted in Fig. 2, since electron-phonon interactions, ignored 
in our calculations, can have a strong effect on the energy of the PP2 absorption. The relative energies of these experimental PAs can perhaps also be dependent on chain length, and hence may be more strongly conformation-dependent than the lower energy PAs. This may explain the difference in the conclusions regarding the energy location of $\mathrm{P}_{2}$ between references 12 and 13. Work is currently in progress to include electron-phonon interactions in our PA calculations to probe this aspect further.

\section{Acknowledgments}

S.M. acknowledges many stimulating discussions with Professors L. J. Rothberg and Z. V. Vardeny that led to new insights. This work was supported by NSF-DMR-0705163.

1 L. Rothberg, in Semiconducting Polymers: Chemistry, Physics and Engineering, Vol. I, Edited by G. Hadziioannou and G.G. Malliaras (John Wiley, 2006), pp. 179-204 and references therein.

2 V. I. Arkhipov and H. Bässler, Phys. Stat. Sol.(a) 201, 1152 (2004), and references therein.

3 B. J. Schwartz, Annu. Rev. Phys. Chem. 54, 141 (2003), and references therein.

4 E. M. Conwell in Organic Eletronic Materials: Conjugated Polymers and Low Molecular Weight Solids, edited by R. Farchioni and G. Grosso (Springer 2001), pp. 127-180 and references therein.

5 I. D. W. Samuel, G. Rumbles and C. J. Collison, Phys. Rev. B 52, R11573 - R11576 (1995).

6 I. D. W. Samuel, G. Rumbles, C. J. Collison, S. C. Moratti and A. B. Holmes, Chem. Phys. 227, 75 (1998).

7 P. K. H. Ho, J. -S. Kim, N. Tessler and R. H. Friend, J. Chem. Phys. 115, 2709 (2001).

8 S.-H. Lim, T. G. Bjorklund, K. M. Gaab and C. J. Bardeen, J. Chem. Phys. 117, 454 (2002).

9 P. J. Brown, D. S. Thomas, A. Köhler, J. S. Wilson, J. -S. Kim, C. M. Ramsdale, H. Sirringhaus and R. H. Friend, Phys. Rev. B 67, 064203 (2003).

10 A. B. Koren, M. D. Curtis, A. H. Francis and J. W. Kampf, J. Am. Chem. Soc. 125, 5040 (2003).

11 J. Clark, C. Silva, R. H. Friend and F. C. Spano, Phys. Rev. Lett. 98, 206406 (2007).

12 C.-X. Sheng, M. Tong, S. Singh and Z. V. Vardeny, Phys. Rev. B 75, 085206 (2007).

13 S. Singh, T. Drori and Z.V. Vardeny, Phys. Rev. B 77, 195304 (2008). 
14 B. Schweitzer, V. I. Arkhipov and H. Bässler, Chem. Phys. Lett. 304, 365 (1999).

15 D. Hertel, Y. V. Romanovskii, B. Schweitzer, U. Scherf and H. Bässler, Synth. Metals 116, 139 (2001).

16 Y. Zhang, Z. Wang, M.-K. Ng and L. J. Rothberg, J. Phys. Chem. B 111, 13211 (2007).

17 V. Moranti, M. Galli, F. Marabelli and D. Comoretto, Phys. Rev. B 79, 045202 (2009).

18 J. Cornil, D. A. dos Santos, X. Crispin, R. Silbey and J. L. Brédas, J. Am. Chem. Soc. 120, 1289 (1998).

19 F. C. Spano, J. Chem. Phys. 122, 234701 (2005).

20 W. Barford, J. Chem. Phys. 126, 134905 (2007).

21 Z. Wang, S. Mazumdar and A. Shukla, Phys. Rev. B 78, 235109 (2008).

22 R.J. Buenker and S.D. Peyerimhoff, Theor. Chim. Acta 35, 33 (1974).

23 P. Tavan and K. Schulten, Phys. Rev. B 36, 4337(1987).

24 A. C. Morteani, P. Sreearunothai, L. M. Herz, R. H. Friend and C. Silva, Phys. Rev. Lett. 92, $247402(2004)$.

25 P. Sreearunothai, A. C. Morteani, I. Avilov, J. Cornil, D. Beljonne, R. H. Friend, R. T. Phillips, C. Silva and L. M. Herz, Phys. Rev. Lett. 96, 117403 (2006).

26 T. Drori, C.-X. Sheng, A. Ndobe, S. Singh, J. Holt, Z. V. Vardeny, Phys. Rev. Lett. 101, 037401 (2008).

27 R. S. Mulliken, J. Am. Chem. Soc. 74, 811 (1952).

28 A. Shukla, H. Ghosh and S. Mazumdar, Phys. Rev. B 67, 245203 (2003).

29 R. Pariser and R. G. Parr, J. Chem. Phys. 21, 466 (1953)

30 J. A. Pople, Trans. Faraday Soc. 49, 1375 (1953).

31 M. Chandross and S. Mazumdar, Phys. Rev. B 55, 1497 (1997).

32 K. Ohno, Theor. Chim. Acta 2, 219 (1964).

33 S. V. Frolov, Z. Bao, M. Wohlgenannt and Z. V. Vardeny, Phys. Rev. Lett. 85, 2196 (2000).

34 S. V. Frolov, Z. Bao, M. Wohlgenannt and Z. V. Vardeny, Phys. Rev. B 65, 205209 (2002).

35 H. Marciniak, M. Fiebig, M. Huth, S. Schiefer, B. Nickel, F. Selmaier and S. Lochbrunner, Phys. Rev. Lett. 99, 176402 (2007).

36 G. Dicker, M. P. de Haas, L. D. A. Siebbeles, and J. M. Warman, Phys. Rev. B 70, 045203 (2004).

37 E. Hendry, M. Koeberg, J. M. Schins, H. K. Nienhuys, V. Sunström, L. D. A. Siebbeles and M. 
Bonn, Phys. Rev. B 71, 125201 (2005).

38 Z. V. Vardeny, private communications.

39 P. B. Miranda, D. Moses and A. J. Heeger, Phys. Rev. B 64, 081201 (R) (2001) 